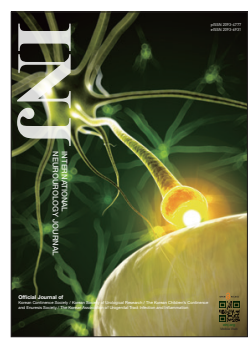

\title{
Importance of Central Regulation for Lower Urinary Tract Functions
}

\author{
Young Sam Cho (iD https://orcid.org/0000-0002-2966-7971 \\ Department of Urology, Kangbuk Samsung Hospital, Sungkyunkwan University School of Medicine, Seoul, Korea \\ E-mail: choys1011@naver.com
}

Lower urinary tract function depends on the coordinated activity of the bladder and the urethral sphincter. Normal lower urinary tract function is under the control of a complex neural network that involves the central and peripheral nervous system [1]. There are well-known structures that play an important role in the control of the lower urinary tract, such as the insula, the anterior cingulate cortex, the prefrontal cortex, and the periaqueductal grey (PAG) during urinary storage, and the medial prefrontal cortex, the hypothalamus, the PAG, and the pontine micturition center during voiding [2].

Lower urinary tract dysfunction (LUTD) is a common consequence of neurological diseases, and the patterns of bladder storage and voiding dysfunction are influenced by the level of the neurological lesion. For example, suprasacral spinal cord or suprapontine lesions result in detrusor overactivity, which is characterized by involuntary contractions of the bladder wall. Additionally, lesions of the spinal cord may result in detrusorsphincter dyssynergia, a simultaneous contraction of the detrusor and the sphincters during attempted voiding [3].

Many neurological diseases affecting the brain, the spinal cord, and the peripheral nervous system are associated with various LUTDs. This is a very well-known fact, and urologists are familiar with it. Typical examples of such diseases include cerebral palsy, stroke, head injury, multiple sclerosis, Parkinson disease, dementia, spinal cord injury, and peripheral neuropathy. However, the association between central regulation of micturition and epilepsy has been comparatively less investigated and less well-known.

In the present issue of the International Neurourology Journal (INJ), a review of central micturition regulation and its association with epilepsy focuses on the neurophysiological mechanisms and their disruption during seizures [4]. The fact that micturition can trigger the onset of seizures raises the following discussion points. First, the question arises whether we know enough about the role of the central nervous system in LUTD. I completely agree with the authors' opinion that our current knowledge of LUTD is limited to an identification of some major brain regions in a few common pathologies. Secondly, the limited understanding of all central nervous mechanisms controlling micturition is likely to be related to the limited number of treatment options in LUTD. So far, the main treatment methods in LUTD such as drugs or neuromodulation are predominantly target the peripheral nervous system. Therefore, it is very important to conduct more research and develop further our knowledge about the central regulation of micturition. These efforts may help us develop new treatments as well as prevention strategies for LUTD.

- Conflict of Interest: No potential conflict of interest relevant to this article was reported.

\section{REFERENCES}

1. Fowler CJ. Autonomic disorders of the urogenital system. CONTINUUM: Lifelong Learn Neurol 2007;13:165-81.

2. Fowler CJ, Griffiths D, de Groat WC. The neural control of micturition. Nat Rev Neurosci 2008;9:453-66.

3. Tudor KI, Sakakibara R, Panicker JN. Neurogenic lower urinary tract dysfunction: evaluation and management. J Neurol 2016;263: 2555-64.

4. Jang HJ, Kwon MJ, Cho KO. Central regulation of micturition and its association with epilepsy. Int Neurourol J 2018;22:2-8. 